\title{
Experimental and numerical modeling for flattening and rapid solidification with crystallization behavior of supersonic ceramic droplets
}

\section{Y. Wang}

Xi'an Jiaotong University

Y Bai ( $\sim$ byxjtu@mail.xjtu.edu.cn )

Xi'an Jiaotong University

$\mathrm{K} \mathrm{Wu}$

Xi'an Jiaotong University

$J$ Zhou

Xi'an Jiaotong University

M G Shen

Xi'an Jiaotong University

$Y$ Ming

Hefei General Machinery Research Institute

Q. Liu

Xi'an Jiaotong University

N J Chong

Xi'an Jiaotong University

Y W Sun

Xi'an Jiaotong University

Y B Hu

Xi'an Jiaotong University

X J Du

Xi'an Qinghua Company North Special Energy Group Co.Ltd

Z B She

Xi'an Qinghua Company North Special Energy Group Co. Ltd

\section{Research Article}

Keywords: Supersonic droplets, Refinement, Numerical model, Multiphase flow, Grain growth

Posted Date: September 21st, 2020

DOl: https://doi.org/10.21203/rs.3.rs-79503/v1 
License: (c) (i) This work is licensed under a Creative Commons Attribution 4.0 International License. Read Full License 


\title{
Experimental and numerical modeling for flattening and rapid solidification with crystallization behavior of supersonic
}

\section{ceramic droplets}

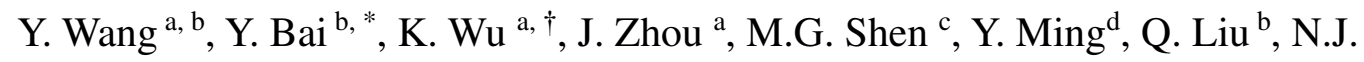 \\ Chong ${ }^{\text {b }, ~ Y . W . ~ S u n ~}{ }^{\text {b }, ~ Y . B . ~ H u ~}{ }^{\text {b }}$ X.J. Du ${ }^{\mathrm{e}}$, Z.B. She $\mathrm{S}^{\mathrm{e}}$ \\ a State Key Laboratory of Electrical Insulation and Power Equipment, Xi'an Jiaotong University,
} Xi'an, 710049, PR China

b State Key Laboratory for Mechanical Behavior of Materials, Xi'an Jiaotong University, Xi'an, 710049, PR China

${ }^{c}$ State Key Laboratory for Manufacturing Systems Engineering, Xi'an Jiaotong University, Xi'an, 710049, PR China

${ }^{\mathrm{d}}$ Hefei General Machinery Research Institute, Hefei, 230031, PR China

e Xi'an Qinghua Company North Special Energy Group Co. Ltd. Measurement and Physical and Chemical Center, Xi'an, 710025, PR China

\begin{abstract}
:
Successive impingement of supersonic droplets after refining in plasma jet usually forms a fine-lamellar structured coating with high mechanical properties. However, the comprehensive process (such as flattening, rapid solidification and crystallization) of high-velocity impact of refined droplets is difficult to understand. In this study, an experimental study showed that the content of refinement droplets reached to $90 \%$ and displayed the multi-scale equiaxed grains morphology at extremely rapid solidification rate. Phase-field model revealed a hybrid coalescence
\end{abstract}

\footnotetext{
${ }^{*}$ Corresponding author. E-mail: byxjtu@ mail.xjtu.edu.cn

${ }^{\dagger}$ Corresponding author. E-mail: wukai@mail.xjtu.edu.cn
} 
growth of oriented attachment and migration of grains boundary under the dynamic temperature gradient. Furthermore, an optimized numerical model that consisted of the Navier-Stokes and energy balance equations coupled with the Cahn-Hilliard and phase-field model for growth orientation of grains was developed to accurately reproduce the comprehensive process of refined supersonic droplets. The size distribution and crystallographic orientation of columnar grains for single or two flattened droplets were in a good agreement with the experimental results. The interface between two-flattened droplets exhibited an epitaxial growth of columnar grains. This optimized model can be an effective method in predicting the flattening and solidification with crystallization behavior of droplets during plasma spraying.

Keywords: Supersonic droplets; Refinement; Numerical model; Multiphase flow; Grain growth

\section{Introduction}

The widely used industrial manufacturing processes usually involve the growth of single crystal, metal additive manufacturing, laser-assisted thin film and thermal spray coating [1-2]. Generally, thermal spray process can be realized by rapidly quenching techniques. Due to the non-equilibrium driving effects, it is possible to produce supersaturated solid-solutions, quasi-crystals, metastable crystalline, amorphous and grain-refined materials [3-4]. Thermal sprayed coating is associated with the rapid solidification and crystallization behavior of flattened droplets [5-7]. These flattened droplets (splats) can serve as the building blocks of coating, which consists of numerous columnar grains that are originated from the heterogeneous nucleation of droplet and competitive growth under the thermal flow direction [8]. In addition, the crystallographic orientation of columnar grains is another important factor that affects the shape of grains. Some researchers showed that thermal 
expansion coefficient and Young's modulus of splat were closely related to crystallographic orientation [9]. The crystallographic orientation is determined by the solidification of droplet [10-11]. During plasma spraying, the solidification rate of ceramic droplet is approximately $10^{4} \sim 10^{6} \mathrm{~K} / \mathrm{s}$. For a metal droplet, the solidification rate is up to $10^{6} \sim 10^{8} \mathrm{~K} / \mathrm{s}$ [12]. Our previous study suggested that the average height and width of columnar grains presented submicron or nano-scale crystallographic texture when the solidification rate of a ceramic supersonic in-flight droplet drastically increased to $10^{9} \mathrm{~K} / \mathrm{s}$ [13-14]. The rapid solidification rate resulted in the formation of fine-lamellar structured coating with multi-scale crystallographic texture, which exhibited the excellent comprehensive performance (e.g. bonding strength, heat temperature resistance, anti-oxidation and thermal shock resistance) [15-17].

Although the crystallographic orientation can be studied by a situ heating HRTEM technology $[11,13]$, the dynamic growth behavior of multi-scale grains of ceramic droplet is still hardly observed by some experiment methods. A physical insight into the growth and formation process of multi-scale grains within finelamellar structure at extremely rapid solidification is great of importance and necessary. Phase-field method is referred to as a diffuse interface method. This method is initially developed by Landau (1986) for the investigation of non-linear transition phenomena of materials [18]. The phase-field equation is based on the nonlinear dynamics theory and can provide a useful mathematical framework [19]. Over the past three decades, this method has been adopted to simulate the formation and evolution of microstructure (e.g. dendritic and eutectic growth) during the undergoing solidification or phase transformation of materials [20-21]. On account of the category of moving boundary problems, the liquid-grain-grain triple junction and stabilized widths of intercalating phases at grains boundaries were studied [22-23]. Furthermore, some researchers also took into account the orientation behavior of anisotropic growth 
of multi-scale grains and subsequent formation of grain boundary after solidification [24-26]. Nevertheless, the current phase-field models usually ignore the compatibility between the model parameter and physical phenomena. The collision and growth mode of multi-scale grains at extremely rapid solidification rate are still not fully understood for one supersonic droplet impacting on a substrate.

The impacting phenomenon of a supersonic droplet involves the coupling multiphysics. Cahn and Hilliard [27] proposed the use of the phase-filed model to solve the problem of phase separation. Subsequently, Jacqmin [28] reported the combination of Cahn-Hilliard and Navier-Stokes equations to apply in the gas-liquid two-phase flow, and derived the surface tension expression in the phase field. Pasandideh-Fard and P. Wei et al [29-30] established the mathematical model of the flattening and solidification processes, which was known as the phase transformation CSLVOF model from liquid phase to solid phase. However, the method is needed to track the free interface and is unable to describe the nucleation and dynamic growth of the grains within solidified splats. Recently, a coupled CFD and diffuse interface model has been developed to reproduce the solidification microstructure formation of supersonic YSZ droplet in our previous works [31-33]. Nevertheless, due to in-flight droplets melted and broke up through strong coupling with the supersonic plasma jet in thermal and momentum transfer, the supersonic droplets were found a significant size refinement and had extremely rapid solidification rate, which led to the formation of fine-lamellar structured coatings [34]. The flattening morphology and crystallization texture of single or sequential droplets after refining in supersonic plasma jet are not validated systematically.

Based on the above background, in the present work, an experimental study was carried out in order to study the refinement of YSZ droplets and crystallization texture. Furthermore, an optimized numerical model accurately reproduced the coupling 
multi-physics process after impact of supersonic refined droplets. The relationship between the solidification rate and crystallographic orientation of grains is elaborated in order to clarify the underlying growth mechanism of equiaxed or columnar grains of flattened supersonic droplets at an extremely rapid solidification rate.

\section{Experimental and mathematical methods}

\subsection{Materials and experimental methods}

Two methods were employed to collect the supersonic droplets via a water bath and a V-shaped shield. The experimental set-up is shown in Fig. 1. The corresponding coating was deposited by a high efficiency supersonic atmospheric plasma spraying (SAPS) system. The temperature and velocity of in-flight droplets were measured by Spray Watch $2 \mathrm{i}$ online monitoring system (Oseir, Finland). Meanwhile, the spraydried and sintered $\mathrm{ZrO}_{2}-8 \mathrm{wt} \% \mathrm{Y}_{2} \mathrm{O}_{3}$ (YSZ) powders were used as the starting feedstock materials. The feedstock powder after sieving had a near-perfect spherical morphology with a particle size of $25-35 \mu \mathrm{m}$ and the average size was approximately $30 \mu \mathrm{m}$. The grain size of the feedstock powder was in the range of 100 to $900 \mathrm{~nm}$, as shown in Fig. 2a-c [35-36]. The morphology of collected powder in the water bath is shown in Fig. 2d-f. Compared with the feedstock powder as shown in Fig. 2a-c, the size of collected powder in the water bath significantly decreased to $0.5-6 \mu \mathrm{m}$ due to the strong coupling with the supersonic plasma jet in SAPS, which directly cause the fragmentation of feedstock powder. Meanwhile, the grain size of collected powder greatly reduced to $100 \sim 200 \mathrm{~nm}$.

\subsection{Specimen characterization}

In order to further reveal the grain boundaries, phases and other microstructural details, the specimens were thermally etched. The thermal etching was conducted at $1250{ }^{\circ} \mathrm{C}$ for $3 \mathrm{~h}$ in a furnace, and then cooled down to the room temperature [35]. The 
morphology of thermal etched coating was observed by scanning electron microscopy (SEM, VEGAII XMU, Tescan, Czech Republic). The growth behavior of grain was analyzed using high resolution transmission electron microscopy (HRTEM, JEM2100F, JEOL, Japan). Plan-view and cross-section specimen for HRTEM analysis were prepared by mechanical grinding, polishing and dimpling, followed by Ar-ion milling using a Gatan 691 precision ion polishing system (Gatan Inc., USA). The HRTEM was equipped with an X-twin objective lens with coefficient of spherical aberration $\mathrm{Cs}=1.0 \mathrm{~mm}$ (point to point resolution of $0.23 \mathrm{~nm}$ ) and operated at $200 \mathrm{kV}$ accelerating voltage. Besides, the grain size and preferred orientation of specimen were further characterized by EBSD (Oxford, UK). The EBSD measurement was performed on the surface specimen at the acceleration voltage of $15 \mathrm{kV}$ and studied the final crystallographic texture that formed by the flattening and solidification behavior of in-flight droplets.

\subsection{Mass, momentum, energy and phase field equations of droplet impact}

A supersonic in-flight droplet after impacting on the substrate is a complex fluid flow, heat transfer, solidification, nucleation and oriented growth of grains [32-33]. In order to accurately reproduce this process, the droplet flow and solidification are firstly analyzed, and then added the nucleation and oriented growth of grains. For the fluid flow during flattening, a phase field method is used to describe the evolution of gas-liquid interface. The fixed grids are directly handled a moving free surface as encountered in YSZ droplet spreading. The phase field parameter $c$ is distinguished into the liquid $(c=0)$ and gas phase $(c=1)$. The Cahn-Hilliard equation will be illustrated the evolution of $c$, as follows:

$$
\begin{aligned}
& c_{t}+\mathbf{u} \cdot \nabla c-M \nabla^{2}(\delta f / \delta c)=0 \\
& f(c)=\frac{1}{2} \xi \gamma \alpha|\nabla c|^{2}+\xi^{-1} \gamma \alpha \cdot \frac{1}{4} c^{2}(1-c)^{2}
\end{aligned}
$$


where $\mathbf{u}$ is velocity, $M$ the diffusion strength, $\delta f / \delta c$ the chemical potential, $f(c)$ the bulk free energy density, $\xi$ the interfacial thickness, $\gamma$ surface tension, $a$ a constant $(a=6 \sqrt{2})$. The system energy finally reaches to an equilibrium statue by the equation of $F=\int f(c) d V$.

The primary variable is velocity and pressure in the flow field. For the incompressible Newtonian fluids of YSZ droplet, the mass and momentum equations are as follows:

$$
\begin{aligned}
& \nabla \cdot \mathbf{u}=0 \\
& \frac{\partial \mathbf{u}}{\partial t}+\mathbf{u} \cdot \nabla \mathbf{u}=-\frac{\nabla p}{\rho(c)}+\frac{\nabla \cdot \sigma}{\rho(c)}+\mathbf{g}+\frac{G \nabla c}{\rho(c)}+S
\end{aligned}
$$

where $p$ is the mechanical pressure, $\sigma=\mu(c)\left(\nabla \mathbf{u}+\nabla \mathbf{u}^{T}\right)$ Newtonian stress tensor, g local gravity acceleration, $G=\delta f / \delta c$ the chemical potential, $G \nabla c / \rho(c)$ denotes the gas-liquid surface tension. The thermophysical properties of YSZ materials are identified as the function of phase field parameter $c$. The expression of density and dynamic viscosity are $\rho(c)=\left(\rho_{g}-\rho_{l}\right) c+\rho_{l} \quad$ and $\quad \mu(c)=\left(\mu_{g}-\mu_{l}\right) c+\mu_{l}$, respectively. It's worth noting that the thermal source $S$ in the Eq. (4) promotes the nucleation and oriented growth of grains when solidification of YSZ droplet. It is expressed as $S=-d\left[\left(1-F_{l}\right)^{2} /\left(F_{l}^{3}+b\right)\right] \cdot \mathbf{u}$. The liquid fraction of thermal source is equivalent to $F_{l}=(1-\phi) / 2$. One constant $b$ is 0.001 , and other $d=10^{9}$ represents the convection heat transfer in the mushy zone.

For the heat transfer with phase change during solidification, an energy equation is required and shown in Eq. (5). It is related to the surface temperature and in-flight velocity of YSZ droplet. Moreover, some thermophysical properties of YSZ materials are taken into account in Eq. (5), including density, specific heat and thermal 
conductivity. Specially, $(\partial \phi / \partial t) \cdot\left(\rho_{l} L_{l} / 2\right)$ in the Eq. (5) means the release of latent heat. When phase field parameter $c>0.5$, the $\partial \phi / \partial t$ is equaled to zero. When $c \leq 0.5$, the $\partial \phi / \partial t$ is updated.

$$
\rho(c) \cdot c_{p}(c) \cdot[(\partial T / \partial t)+\mathbf{u} \cdot \nabla T]=\nabla \cdot[k(c) \nabla T]-(\partial \phi / \partial t) \cdot\left(\rho_{l} L_{l} / 2\right)
$$

\subsection{Phase field equation for crystallization behavior}

For the crystallization behavior when solidification, a phase-field model is employed to study this physical process. The free energy of system is given by:

$$
F=\int d V\left[f(\phi, u)+\frac{\varepsilon_{\phi}^{2}(\varphi)}{2}|\nabla \phi|^{2}+s h(\phi)|\nabla \phi|+\frac{\varepsilon_{\theta}^{2}}{2} h(\phi)|\nabla \phi|^{2}\right]
$$

where $f(\phi, u)$ is the bulk free energy density [16]. $\varepsilon_{\phi}(\varphi)$ is the anisotropic gradient energy coefficient. The second term accounts for the energy penalty within the interface. The latter terms ensure the motion and stable of grain boundaries. $s$ and $\varepsilon_{\theta}$ specify the strength of the coupling between $\phi$ and $\nabla \phi . \varphi$ is the inclination angle of solid-liquid interface $(\mathbf{n}=\nabla \phi /|\nabla \phi|)$ with respect to the $x$ axis, which defined as $\varphi=\tan ^{-1}(\partial \phi / \partial y) /(\partial \phi / \partial x)$

Based on the bulk free energy density $[25,36]$, the kinetic evolution of solidliquid order parameter $(\phi)$ ranging from the liquid $(\phi=-1)$ to the solid phase $(\phi=1)$ is described by as follows:

$$
\begin{array}{r}
\tau_{\phi}(\varphi) \frac{\partial \phi}{\partial t}=\left(1-\phi^{2}\right)\left[\phi-\lambda u\left(1-\phi^{2}\right)\right]+\nabla \cdot(\Lambda \cdot \nabla \phi) \\
-2 s(1+\phi)|\nabla \theta|-\varepsilon_{\theta}^{2}(1+\phi)|\nabla \theta|^{2}
\end{array}
$$

where $\tau_{\phi}(\varphi)$ is the interface attachment kinetic times. $\lambda$ specifies the coupling between phase field and diffusion fields. $u=\left(T-T_{m}\right) /\left(L / C_{p}\right)$ refers to the dimensionless temperature. $T_{m}, L, C_{p}$ are melting temperature, latent heat and 
specific heat capacity, respectively. Particularly, the $-2 s(1+\phi)|\nabla \phi|$ in the Eq. (7) prompts the stable of grain boundaries, $-\varepsilon_{\theta}{ }^{2}(1+\phi)|\nabla \theta|^{2}$ represents the rotation and migration of grain boundaries during solidification.

In addition, the orientational field of grains growth for $\theta$ is given by:

$$
P \tau_{\theta}(1+\phi)^{2} \frac{\partial \theta}{\partial t}=\nabla \cdot\left[(1+\phi)^{2}\left(\frac{s}{|\nabla \theta|}+\varepsilon_{\theta}^{2}\right) \cdot \nabla \theta\right]
$$

where $P$ is the inverse mobility function. $P\left(\varepsilon_{\theta}|\nabla \theta|\right)=1+\left(\mu / \varepsilon_{\theta}-1\right) e^{-\beta \varepsilon_{\theta}|\nabla \theta|} \cdot \mu$ represents grain rotation. $\beta$ is grain boundary mobility. $\boldsymbol{\Lambda}$ is a second order tensor and is given by as follows:

$$
\boldsymbol{\Lambda}=\left[\begin{array}{cc}
\varepsilon_{\phi}^{2} & -\varepsilon_{\phi} \cdot\left(\partial \varepsilon_{\phi} / \partial \varphi\right) \\
\varepsilon_{\phi} \cdot\left(\partial \varepsilon_{\phi} / \partial \varphi\right) & \varepsilon_{\phi}^{2}
\end{array}\right]
$$

Actually, the crystal growth is accompanied by the anisotropy feature. $\varepsilon_{\phi}(\varphi)$ and $\tau_{\phi}(\varphi)$ are used to describe this anisotropic gradient energy and can be described by $\quad \varepsilon_{\phi}(\varphi)=\varepsilon_{\phi}\left\{1+\varepsilon_{4} \cos [4(\varphi-\phi)]\right\} \quad$ and $\quad \tau_{\phi}(\varphi)=\operatorname{t}_{\phi}\left\{1+\varepsilon_{4} \cos [4(\varphi-\phi)]\right\}^{2}$, respectively.

\subsection{Boundary conditions}

For computation of fluid motion and phase transformation, the substrate is excluded. For computation of heat transfer, the two-phase flow region and the substrate is combined with all boundaries adiabatic. The setting up of boundary conditions is shown in Fig. 3a. The order parameter $c$ at the substrate surface satisfies the following equation:

$$
\xi \gamma \alpha \cdot \mathbf{n} \cdot \nabla c+f_{w}^{\prime}(c)=0
$$

where $f_{w}(c)$ is the free energy density, and it is expressed as $f_{w}(c)=\left[-\gamma \cos \theta_{s}\left(4 c^{3}-6 c^{2}+1+\gamma_{w 1}+\gamma_{w 2}\right)\right] / 2, \mathbf{n}$ is the unit normal pointing to the 
wall, $f_{w}(c=0)$ gives the liquid-solid interfacial tension $\gamma_{w 1}$ and $f_{w}(c=1)$ produces the gas-solid interfacial tension $\gamma_{w 2}$, the two determining the static contact angle $\theta_{s}$ through Young's equation $\gamma_{w 2}-\gamma_{w 1}=\gamma \cos \theta_{s}$.

\subsection{Numerical procedures}

The set of equations are explicitly discretized using the finite difference method, with upwind scheme to deal with convective terms. Unlike the analytical solution, which gives precise values of variables of interest at each and every point in a domain of interest, the numerical solution provides solutions only at discrete points of the domain. The first step in any numerical analysis must therefore be to select these points. This may be done by subdividing the medium of interest into a number of small regions and assigning to each cell vertex a reference point as in Fig. $3 b$. The exact differential equations will be reduced to algebraic equations after approximating the derivative terms. Besides, a series of ghost nodes, marked in brown and red, render boundary nodes inner ones. No modification is thus to be made to the discretized equations when considering boundary nodes. The no slip condition on the bottom is applied at $\mathrm{j}=1$, while $\mathrm{j}=0$ represents the nodes in the substrate. The thermal contact resistance is applied between row $\mathrm{j}=1$ and $\mathrm{j}=0$. The two order parameters definition of droplet after impacting on a substrate is shown in Fig. 3c. As seen from Fig. 3c, the pair of $c=1$ and $\phi=-1$ represents surrounding atmosphere of in-flight droplet, $c=0$ and $\phi=-1$ means in-flight droplet, $c=0$ and $\phi=1$ is the grains when solidification. Meanwhile, the time step of computation process is set as the order of $10^{-13} \mathrm{~s}$. During a computational time interval, computation starts from the evolution of $\phi$ and $\theta$, which is confined to the region with $\mathrm{c} \leqslant 0.5$, then proceeds to heat transfer and fluid motion, thus completing one loop. In order to accelerate computation, a parallel programing based on OpenMP is applied in this work. 


\section{Results and Discussion}

\subsection{Refinement of a supersonic ceramic droplet}

The microstructure and property of an as-sprayed coating are highly dependent on flattening and solidification behavior of in-flight droplets. The spray parameters and corresponding temperature and velocity of droplets are listed in Table 1. As seen from Table 1, owing to a Laval nozzle with an internal injection port, the temperature and velocity of supersonic droplets increased with the increase of spray power. The flattened droplets (splats) gradually converted from net-shaped into finger-shaped splashing morphology in Fig. 4a-c. Our previous study showed that the net-shaped splat was multilayer and finger-shaped one was monolayer structure, respectively [14]. Furthermore, as seen from Fig. 4a-c, some refined splats formed and located in the periphery of splashing morphology. However, the corresponding structure was obviously evolved into disk-shaped one. Based on the isovolumetric feature of both spherical droplets and splats, the diameter of original droplet refined splats was calculated and less than $5 \mu \mathrm{m}$ through a 3D Laser Scanning Microscope. After statistical analysis, the content of fragmented droplets with different temperatures or velocities was $75.16 \%, 86.32 \%$ and $90.21 \%$, respectively. These results suggested that the simultaneous improvement of temperature and velocity of in-flight droplets was beneficial to the fragmentation of droplets.

The size reduction and morphology change of splats suggested that the in-flight droplets were melted and broken up by a combined action of forces associated with a supersonic plasma jet, which was dependent on the critical threshold between the hydrodynamic pressure and the added pressure caused by the surface tension force [34]. It was firstly continued to the deformation of droplet without disintegrating in the fragmentized process. When the added pressure force was inferior to the 
hydrodynamic pressure, the fragmentation of droplet occurred. For one spherical droplet, the added pressure can be described by Young-Laplace $\left(\Delta P=4 \sigma_{p} / d_{p}\right)[34$, 37]. The calculated added pressure was $5.7 \times 10^{4} \mathrm{~Pa}$ based on a YSZ droplet with the average size of $30 \mu \mathrm{m}$. Nevertheless, the hydrodynamic pressure provided by drag force was described as follows [38]:

$$
P_{f}=1 / 8 \cdot C_{f} \rho_{p}\left|v_{p}-v_{d}\right|\left(v_{p}-v_{d}\right)
$$

where $P_{f}$ is the drag force, $\rho_{p}$ is the density of plasma jet, $C_{f}$ is the drag coefficient $\left(C_{f}=24 / \operatorname{Re}+6 /(1+\sqrt{\operatorname{Re}})+0.4\right)[39], v_{p}$ is the velocity of plasma jet obtained by CFD-ACE+V2009.0 (ESI GROUP, America) simulation, $v_{d}$ is the in-flight velocity of YSZ droplet obtained by Spray Watch $2 \mathrm{i}$ system and Re is Reynolds number of YSZ droplet $\left(\operatorname{Re}=\left(\rho_{p}\left|v_{p}-v_{d}\right| d_{p}\right) / \mu_{g}\right)[40,41]$. Our previous work showed that the hydrodynamic pressure was significantly larger than the added pressure $\left(5.7 \times 10^{4}\right.$ $\mathrm{Pa}$ ), indicating that the breakup of YSZ droplet occurred in the supersonic plasma jets [34].

\subsection{Grain size distribution and crystallographic orientation of refined droplet}

Based on the content of refined droplet was in the majority $(>90 \%)$ via the above experiments collection in Fig. 4, which was regarded as a typical droplet to study in the following experiments and simulations. Fig. 5 shows the texture and crystallographic orientation of refined droplet after flattening and solidification. The inverse pole figure (IPF) and orientation map of refined droplet are shown in Fig. 5a. As seen from Fig. 5a, the grain size of splat was ranging from $0.05 \mu \mathrm{m}$ to $0.51 \mu \mathrm{m}$. According to the statistical results, the content of grains with size of $0.15 \mu \mathrm{m}$ reached to $14 \%$ and is much higher than other grains. The small-sized grains originated from the rapid solidification rate of supersonic in-flight droplet impacting on a substrate. 
Besides, these grains had a preferential growth along the [001] direction of $\mathrm{X}$ axis. Fig. $5 b$ depicts the surface morphology of coating after thermal etching at $1250{ }^{\circ} \mathrm{C}$ for $3 \mathrm{~h}$. It can be seen from Fig. 5b that most of grain sizes were distributed in the range of 100-200 nm and its percentage was $15 \%$. Additionally, some nano grains were also verified by TEM as shown in Fig. 5c. The selected area electron diffraction (SAED) patterns of nano-grains at the "A" position suggested that the nano-grains belonged to the tetragonal phase with preferential growth along the $[\overline{1} 10]$ direction. The corresponding crystallographic orientation angle $(\theta)$ was $\pi / 4$.

Fig. 6 gives the simulation results of crystallization process and final morphology of equiaxed grains in one flattened YSZ droplet (splat). The average inflight temperature and velocity of YSZ droplet was $(3797 \pm 5) \mathrm{K}$ and $(518 \pm 5) \mathrm{m} / \mathrm{s}$, respectively. The corresponding dimensionless temperature was calculated as 0.59 according to the physical parameter of YSZ materials. These experimental values were considered as the initial conditions and inputted into the model. It was seen from Fig. $6 \mathrm{a}$ and $\mathrm{b}$ that temperature of YSZ droplet gradually decreased during solidification and accompanied by the competitive growth of equiaxed grains. Subsequently, the smaller grains were inclined to larger ones and some obvious thermal interaction zones observed at $t=0.25 \mu \mathrm{s}$. These large-sized grains collided upon each other, resulting in the formation of grain boundaries. Then the motion of grain boundaries occurred during the coarsening and fusion process. With the aim of simulating four-fold anisotropy of equiaxed grains, the crystallographic orientation angle for each solid seed was initially assigned, while it was limited to the scope of $-\pi / 4 \leq \theta \leq \pi / 4[26,42]$. Fig. $6 \mathrm{c}$ shows the grain orientation at the different times. As seen from Fig. 6c, the grain fusion was observed in a relatively low initial orientation mismatch. However, in the case of a large orientation mismatch between 
two impinging grains, the grain boundary was more stable. With the solidification time $(\mathrm{t})$ was sustained up to $0.375 \mu \mathrm{s}$, the grain growth was in a steady state and the final grain size was in the range of $30-120 \mathrm{~nm}$. The above phase field model were further confirmed the accuracy of experimental results.

\subsection{Flattening, solidification and crystallization behavior of refined droplet}

In order to build an integrated and coupling model for one supersonic droplet, the flattening and solidification process are taken into an account. The mesh size is $751 \times 201$ based on a physical domain of $30 \mu \mathrm{m} \times 8 \mu \mathrm{m}$. According to the experimental results, the diameter of fragmented droplet was set to $4.8 \mu \mathrm{m}$ (see Fig. 4). The initial temperature of droplet and preheated substrate were $3000 \mathrm{~K}$ and $423 \mathrm{~K}$, respectively. The contact angle and interfacial heat transfer coefficient at droplet/substrate interface were $120^{\circ}$ and $10^{8} \mathrm{~W} / \mathrm{m}^{2}$. The other thermophysical and model parameters are listed in Table. 2 and 3. Fig. 7 shows the numerical results of droplet with an impact velocity at $518 \mathrm{~m} / \mathrm{s}$. At $0.08 \mu \mathrm{s}$, the flattening process finished. The solidified part simultaneously occurred at the bottom of flattened droplet, suggesting that the impact process was mainly affected by the motion of fluid paralleling to the substrate surface. Besides, the solidification occurred firstly near the impacting point and then expanded as the droplet spread. When the time sustained to $0.2 \mu \mathrm{s}$, the growth rate of columnar grains significantly increased due to the heat transfer energy, which promoted the increase of solidification thickness and eventually led to a stable disk-shaped splat at $0.5 \mu \mathrm{s}$. The flattening ratio (defined as the ratio between the initial diameter and thickness of flattened droplet) was approximately 7.5. Especially, the height and width of columnar grains were and 0.5-0.9 $\mu \mathrm{m}$ and 0.3-0.6 $\mu \mathrm{m}$, respectively.

Fig. 8 depicts the effect of solidification rate on the crystallization texture of columnar grains. The top and bottom boundaries were subjected to fixed temperatures, while others boundaries were adiabatic. The substrate temperature was kept as $1800 \mathrm{~K}$, 
$2000 \mathrm{~K}$ and $2100 \mathrm{~K}$ (see Fig. 8a-c). A group of nuclei with a uniform radius of $10 \Delta x$ were non-equidistantly seeded at the substrate surface. The solidification rate of droplet was calculated by this equation of $\partial T / \partial t \approx \kappa \cdot\left(T_{l}-T_{s}\right) /\left(C_{p} \rho d^{2}\right)[13] . \kappa$ is the thermal conductivity, $T_{l}$ the liquid temperature (i.e. the surface temperature of inflight droplet), $T_{s}$ the substrate temperature. After calculation, the solidification rates of supersonic droplets were approximately $2.3 \times 10^{9} \mathrm{~K} / \mathrm{s}, 2.1 \times 10^{9}$ and $2.0 \times 10^{9} \mathrm{~K} / \mathrm{s}$, respectively. The larger solidification rate as shown in Fig. 8a caused a relatively flat and stable solidification front. When the solidification rate decreased, a typical tip splitting occurred in each grain, leading to the so-called seaweed morphology as shown in Fig. 8b. When the solidification rate further reduced, the dominating crystallographic texture changed into a dendritic shape (see Fig. 8c). This was attributed to the competition between the thermodynamic driving force and surface tension anisotropy, the solidified structure was evolved from a planar shape into a dendritic one. For the columnar grains with a lower solidification rate, it was firstly oriented attachment of grains, and then the migration and junction of grains boundaries under the heat flow direction. The lower heat activated energy could promote the increase of freedom degree of grains rotation. These small-sized grains were inclined to be assimilated by the larger ones through interfacial atom reconfigurations.

When the substrate temperature sharply decreased to $423 \mathrm{~K}$, the simulation results of columnar grains at extremely rapid solidification rate $\left(5.4 \times 10^{9} \mathrm{~K} / \mathrm{s}\right)$ are shown in Fig. 9. It can be seen from Fig. 9a and 9b, the average height and width of columnar grains were $0.6 \mu \mathrm{m}$ and $0.2 \mu \mathrm{m}$, respectively. In order to verify the size of columnar grains, the computational domain of grain growth was a rectangle of $0.6 \times 2$ $\mu \mathrm{m}$ and the initial temperature was linearly distributed along the direction of $\mathrm{y}$ axis. 
When the solidification time was initially $0.01 \mu \mathrm{s}$, these grains shape implied that heterogeneously nucleated grains formed at the bottom of splat and covered the substrate surface. At $t=0.1 \mu \mathrm{s}$, these grains grew directionally to the surface of splat to form a columnar structure. When the flattened droplet was completely solidified ( $t=0.45 \mu \mathrm{s}$ ), the average height and width of columnar grains were $0.6 \mu \mathrm{m}$ and 0.3 $\mu \mathrm{m}$, respectively. The corresponding crystallographic orientation angle was ranging from 0 to $\pi / 4$, which was basically in accordance with above results.

\subsection{Flattening, solidification and crystallization behavior of two refined droplets}

When two successive droplets impinged onto the substrate within a short period of time, the first droplet had been solidified and formed splat/splat interface. The droplet revealed a time-/space-independent feature [43]. In order to further study the thermodynamics and dynamics essence of two droplets, a phase model that can reproduce the flattening and solidification process of two droplets is built and is shown in Fig. 10. The mesh size is $751 \times 351$, corresponding to a physical domain of $30 \mu \mathrm{m} \times 14 \mu \mathrm{m}$. The spreading of second YSZ droplet onto the solidified YSZ splat was similar to the above process of single droplet. But the top surface of solidified splat at $0.4 \mu \mathrm{s}$ was curved, which caused the uprising jet at $0.5 \mu \mathrm{s}$. Besides, the fattening ratio of second droplet was relatively smaller than that of the first one. This was attributed to the reduced thermal diffusion at YSZ splat/splat interface than that of YSZ splat/stainless steel interface. Moreover, due to the same order parameter $\mathrm{c}$ of the Cahn-Hilliard model, a coalescence phenomenon was found at the flattened droplet/splat interface. After $0.5 \mu$ s, the surface tension would pull back of flattened droplet and reduce the surface area. Finally, the splat structure changed into stabilization after $0.8 \mu$ s.

Fig. 11 shows the growth behavior of columnar grains within splat. The corresponding temperature is given in Fig. 12. It was clear that the nucleation 
dynamics between YSZ droplet impacting onto a stainless steel substrate or a YSZ splat are obviously different. In the former, copious heterogeneous nucleation produced at extremely rapid solidification rate $\left(5.4 \times 10^{9} \mathrm{~K} / \mathrm{s}\right)$. However, the irregularity of columnar grains surface in solidified YSZ splat for the latter was served as excellent nucleation catalysts, heterogeneous nucleation seemed much earlier. Due to the heat is transferred into the cool splat, the temperature of first splat dropped under $2500 \mathrm{~K}$ when the second droplet impacted on the first one, which can be demonstrated by the lowering of the isothermal line of $2500 \mathrm{~K}$ at $0.5 \mu$ s (see Fig. 12). Furthermore, all of the nuclei could attain completely coherent interfaces with the underlying grains since the solidifying materials had the same composition and consequently the lattice parameters $[6,12,43,44]$. Such epitaxial growth was also observed in Fig. 11a.

Subsequently, the anisotropy played an important role under thermal driving force, which was demonstrated by the sharp competition of grains at $0.8 \mu \mathrm{s}$. For the temperature distribution in Fig. 12, it was declined rapidly due to around the edge of the bottom splat. Since $0.8 \mu$ s, the spreading of second droplet has terminated. The heat transfer was dominated by thermal conduction. Particularly, as seen from Fig. $11 \mathrm{~b}$, the fluid flow was obviously affected by growth direction of grains at $1.5 \mu$ s. These columnar grains skewed with the lateral flow of droplet spreading. The lateral flow caused grain boundary curve in one direction and surface tension in the opposite direction. The temperature of the second splat reduced below $2500 \mathrm{~K}$ and the number of protrusion continuously decreased due to the accumulation of flattened droplets.

The TEM image of columnar grains as seen from Fig. 11c further suggested the growth mode of columnar grains. The average height and width of columnar grains were $(0.5 \pm 0.1) \mu \mathrm{m}$ and $(0.3 \pm 0.1) \mu \mathrm{m}$, respectively. The growth of columnar grains was highly dependent on the solidification rate of flattened droplet. Our previous 
study showed that the solidification rate of supersonic droplet was approximately $3.9 \times 10^{9} \mathrm{~K} / \mathrm{s}[13,14]$. The preferential growth orientation was [101] direction and the corresponding $\theta$ was considered as $\pi / 4$, which belonged to regular crystallographic orientation angle. At this extremely rapid solidification rate, the average size of equiaxed/columnar grains decreased to a submicron or nano level $[34,35,45]$. The severe non-equilibrium effect promoted the heterogeneous nucleation, leading to the increase of nucleation number. Then the nucleated grains grew laterally until the layer was completely covered. Finally, the competitive growth occurred for the nucleated grains, a stable set of columnar grains continued to solidify and form the preferential orientation through the thickness of the flattened droplet in the opposite direction of the heat flow.

\section{Conclusions}

In this study, based on the refinement droplets in supersonic plasma jet, an experimental and numerical modeling for flattening and rapid solidification with crystallization behavior of supersonic YSZ droplets were studied. The main conclusions are as follows:

(1) The content of refined droplet was about $90 \%$. Simultaneous improvement of temperature and velocity of in-flight droplets was beneficial to the refinement of droplets, which was highly depended on the critical threshold between the hydrodynamic pressure and added pressure caused by the surface tension force.

(2) The crystallographic texture within flattened droplets consisted of submicron/nano equiaxed grains at an extremely rapid solidification $(t=0.375 \mu$ s), which had a hybrid coalescence growth of oriented attachment and migration of grain boundary under the dynamic temperature gradient.

(3) An optimized numerical model that consisted of the Navier-Stokes and energy 
balance equations coupled with the Cahn-Hilliard and phase-field model for growth orientation of grains was developed to accurately reproduce the comprehensive process of refined supersonic droplets.

(4) With the increase of solidification rate $\left(2.0 \times 10^{9} \mathrm{~K} / \mathrm{s} \sim 5.4 \times 10^{9} \mathrm{~K} / \mathrm{s}\right)$, the solidified structure of single flattened droplet was evolved from a dendritic shape into an columnar one. The height and width of columnar grains were basically in accordance with experimental results. For two flattened droplets, their interface exhibited an epitaxial growth of columnar grains.

\section{Acknowledgements}

This work was supported by National Key R\&D Program of China (Grant No.

2018YFB2004002), China Postdoctoral Science Foundation (Grant No. 2019M653598) and Natural Science Foundation of Shaanxi Province (Grant No. 2019TD-020 and No. 2019JQ-586).

\section{References}

[1] Y. Shu, X. Ai, B.Q. Li, Int. J. Numer. Meth. Eng. 69 (2007) 1194.

[2] Y. Zhang, S. Matthews, M. Hyland, Int. J. Heat Mass Tran. 115 (2017) 488.

[3] H. Liu, H.R. Salimi Jazi, M. Bussmann, Acta Mater. 57 (2009) 6013.

[4] J.B. Zhang, H.F. Wang, W.W. Kuang, Acta Mater. 148 (2018) 86.

[5] T. Chraska, A.H. King, Thin Solid Films 397 (2001) 30.

[6] T. Chraska, A.H. King, Thin Solid Films 397 (2001) 40.

[7] C.J. Li, G.J. Yang, C.X. Li, J. Therm. Spray Technol. 22 (2012) 192. 
[8] Z.H. Zhang, J.X. Luo, Q. Li, J. Therm. Spray Technol. 24 (2015) 885.

[9] K. Shinoda, M. Demura, H. Murakami, Surf. Coat. Technol. 204 (2010) 3614.

[10] J.B. Zhang, H.F. Wang, F. Zhang, J. Alloy. Compd. 781 (2019) 13.

[11] X.X. Wei, X. Lin, W. Xu, Acta Mater. 95 (2015) 44.

[12] S.W. Yao, T. Liu, C.J. Li, Acta Mater. 134 (2017) 66.

[13] Y. Wang, Y. Bai, Q.Z. Yang, J. Alloy. Compd. 619 (2015) 820.

[14] Y. Wang, Y. Bai, K. Wu, J. Alloy. Compd. 784 (2019) 834.

[15] Y. Bai, Z.H. Han, H.Q. Li, Surf. Coat. Technol. 205 (2011) 3833.

[16] Y. Bai, L. Zhao, K. Liu, Vacuum, 99 (2014) 119.

[17] J.J. Tang, Y. Bai, J.C. Zhang, J. Alloy. Compd. 688 (2016) 729.

[18] F. Cheng, L.Y. Lian, L.Y. Li, Adv. Sci. 6 (2019) 1802202.

[19] N. Provatas, J.A. Dantzig, B. Athreya, JOM, 59 (2007) 83.

[20] R. Kobayashi, Physica D, 63 (1993) 410.

[21] G. Caginalp, W. Xie, Phys. Rev. E, 48 (1993) 1897.

[22] I. Steinbach, M. Apel, Mater. Sci. Eng. A, 449-451 (2007) 95.

[23] J.A. Warren, R. Kobayashi, A.E. Lobkovsky, Acta Mater. 51 (2003) 6035.

[24] P. Bruna, E. Pineda, J.I. Rojas, J. Alloy. Compd. 483 (2009) 645.

[25] X. Al, Y. Shu, B.Q. Li, Comput. Math. Appl. 52 (2006) 721.

[26] M. Yamaguchi, C. Beckermann, Acta Mater. 61 (2013) 2268. 
[27] J.W. Cahn, J.E. Hilliard, J. Chem. Phys. 28 (1958) 258.

[28] D. Jacqmin, J. Comput. Phys. 155 (1999) 96.

[29] M.P. Fard, V. Pershin, S. Chandra, J. Therm. Spray Technol. 11 (2002) 206.

[30] P. Wei, Z. Wei, S. Li, App. Surf. Sci. 321 (2014) 538.

[31] M.G. Shen, B.Q. Li, Q.Z. Yang, Int. J. Multiphase Flow, 116 (2019) 51.

[32] M.G. Shen, B.Q. Li, Y. Bai, Int. J. Heat. Mass Tran. 150 (2020) 119267.

[33] M.G. Shen, B.Q. Li, Y. Bai, Int. J. Heat. Mass Tran. 157 (2020) 119844.

[34] Y. Bai, L. Zhao, Y. Wang, J. Alloy. Compd. 632 (2015) 794.

[35] Y. Wang, Y. Bai, L. Liu, Appl. Surf. Sci. 363 (2016) 101.

[36] Y. Wang, Y. Bai, T. Yuan, Surf. Coat. Technol. 319 (2017) 95.

[37] R.Q. Duan, S. Koshizuka, O. Yoshiaki, Nucl. Eng. Des. 225 (2003) 37.

[38] Y.P. Chyou, E. Pfender, Plasma Chem. Plasma Proc. 9 (1989) 45

[39] F.M. White, Viscous Fluid Flow, McGraw-Hill, New York, 1974.

[40] S. Kentaro, J. Appl. Phys. 100 (2006) 074903.

[41] C. Josserand, S.T. Thoroddsen, Annu. Rev. Fluid Mech. 48 (2016) 365.

[42] J.K. Ren, Y. Chen, B. Xu, Comp. Mater. Sci. 163 (2019) 37.

[43] E.J. Yang, X.T. Luo, G.J. Yang, Surf. Coat. Technol. 274 (2015) 37.

[44] S.W. Yao, C.J. Li, J.J. Tian, Acta Mater. 119 (2016) 9.

[45] Y. Wang, Y. Bai, K. Wu, Surf. Coat. Technol. 381 (2020) 125115. 

Figures

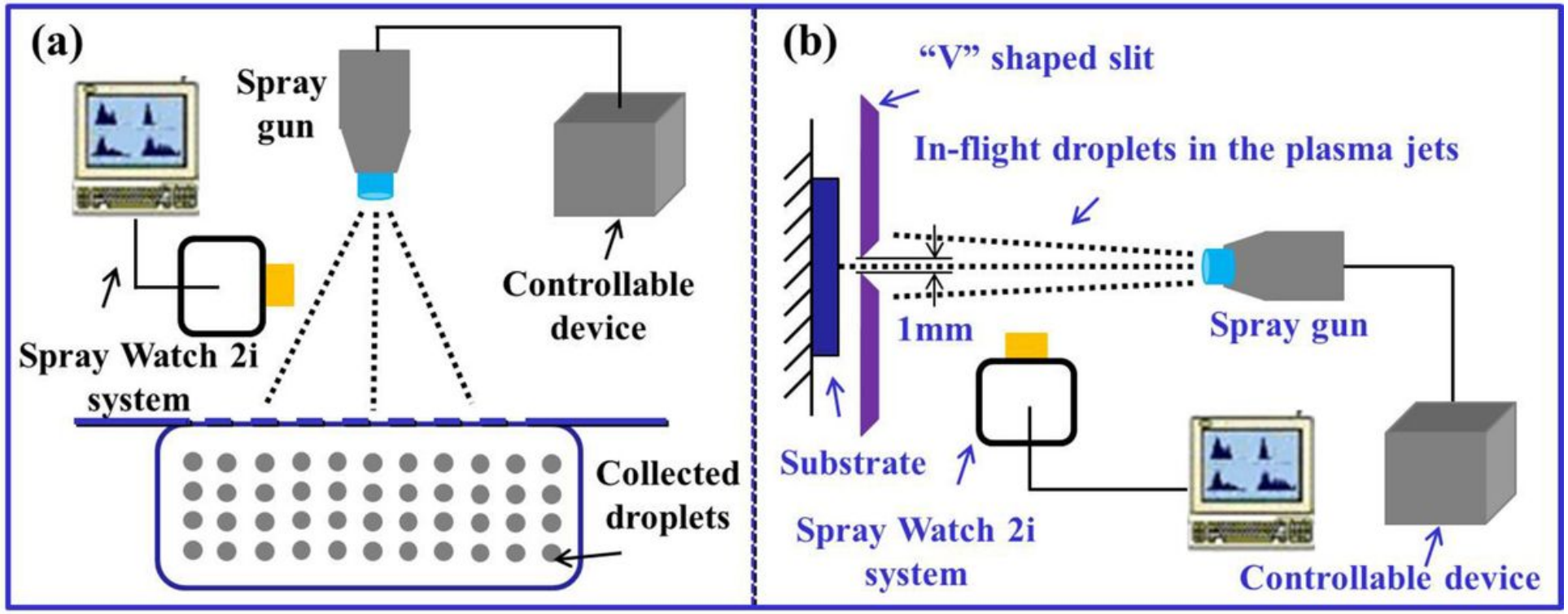

Figure 1

Collection schematic of in-flight droplets via both techniques: (a) experimental set-up of water collection; (b) a V-shaped shield of single-layered flattened droplet.
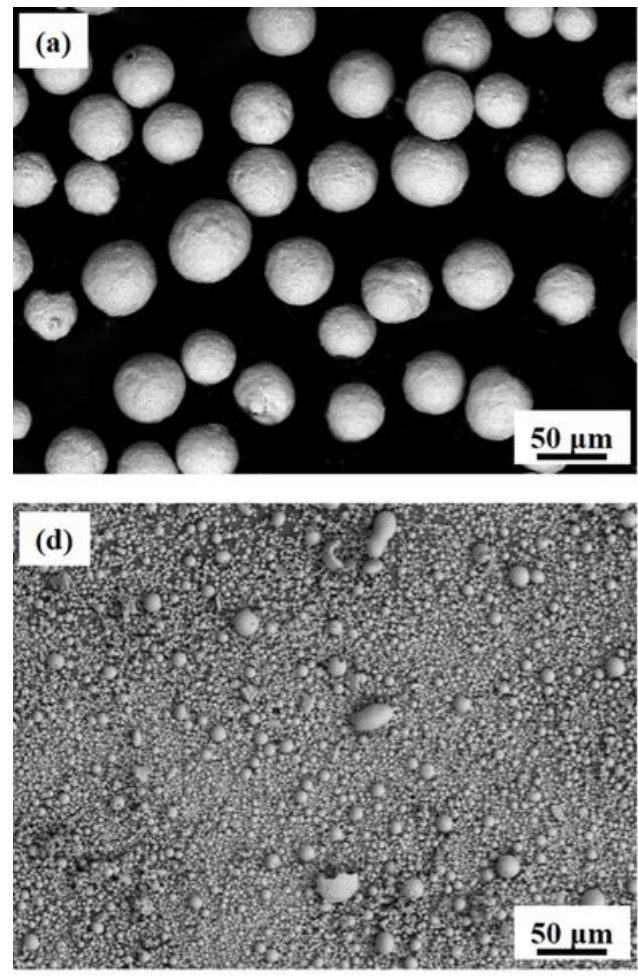
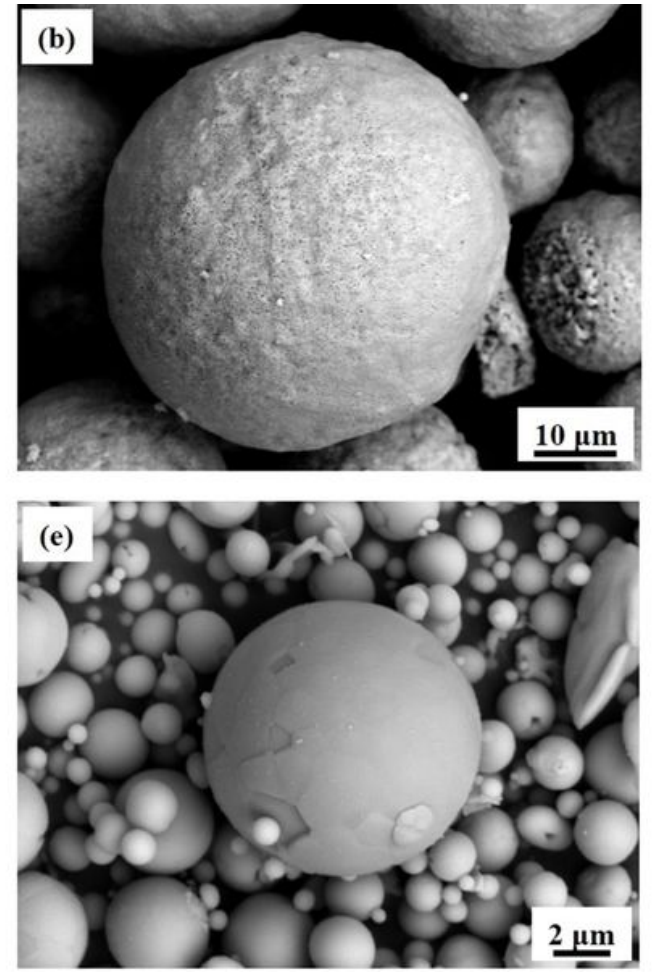
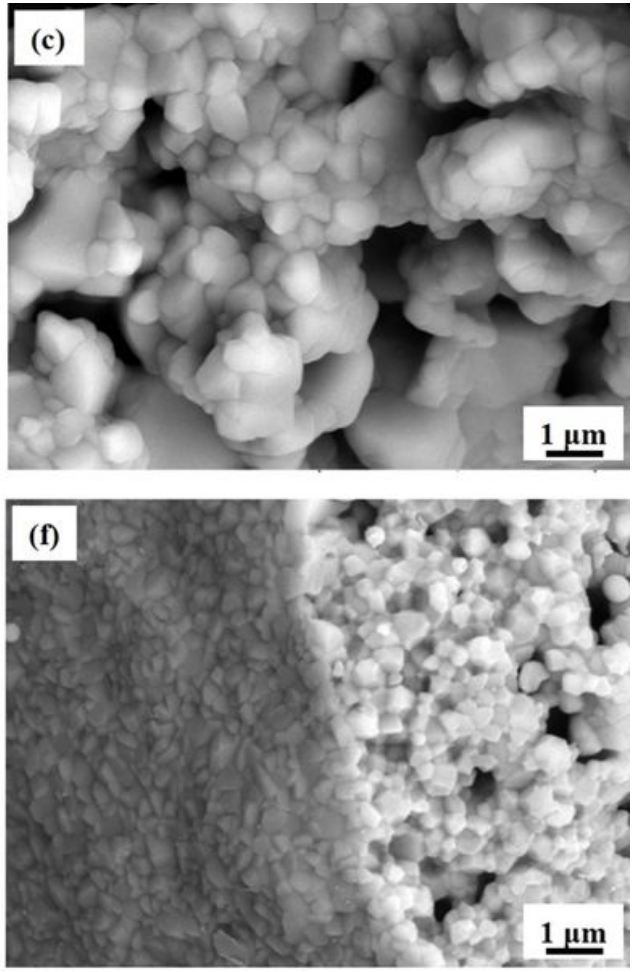

Figure 2

SEM images of powder used for plasma spray: (a) feedstock powder after sieving; (b) high-magnification image of single feedstock powder; (c) grain morphology in the inside of feedstock powder; (d) refinement 
powder morphology after water collection; (e) high-magnification morphology of single refinement powder; (f) grain morphology in the inside of refinement powder.

(a)

(c)

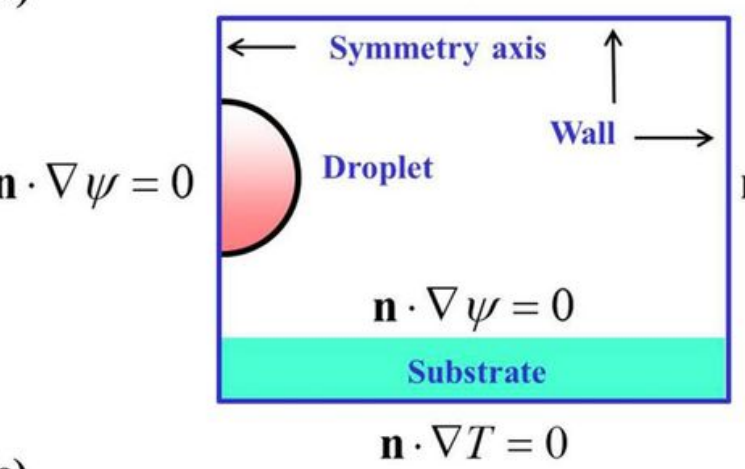

(b)

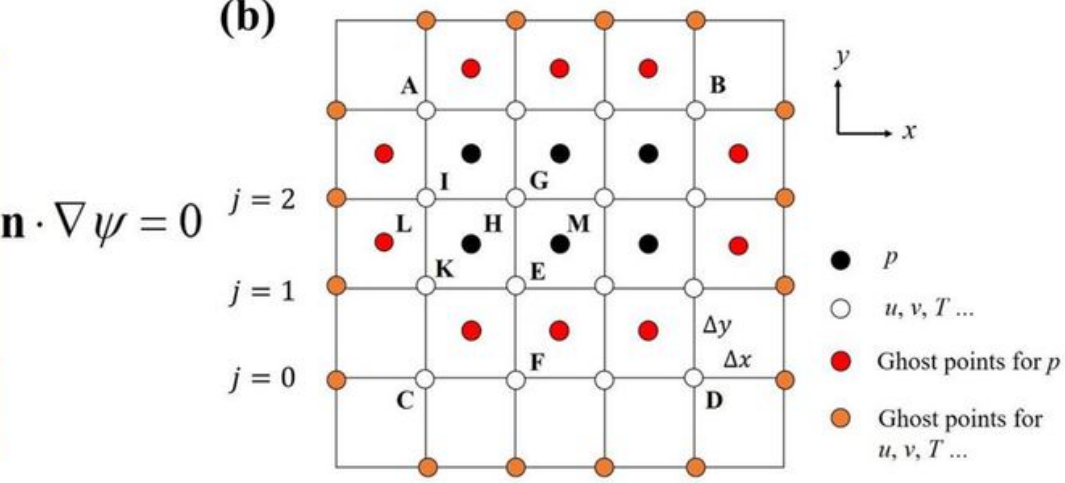

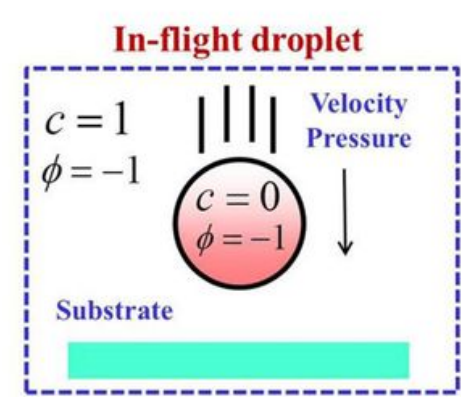
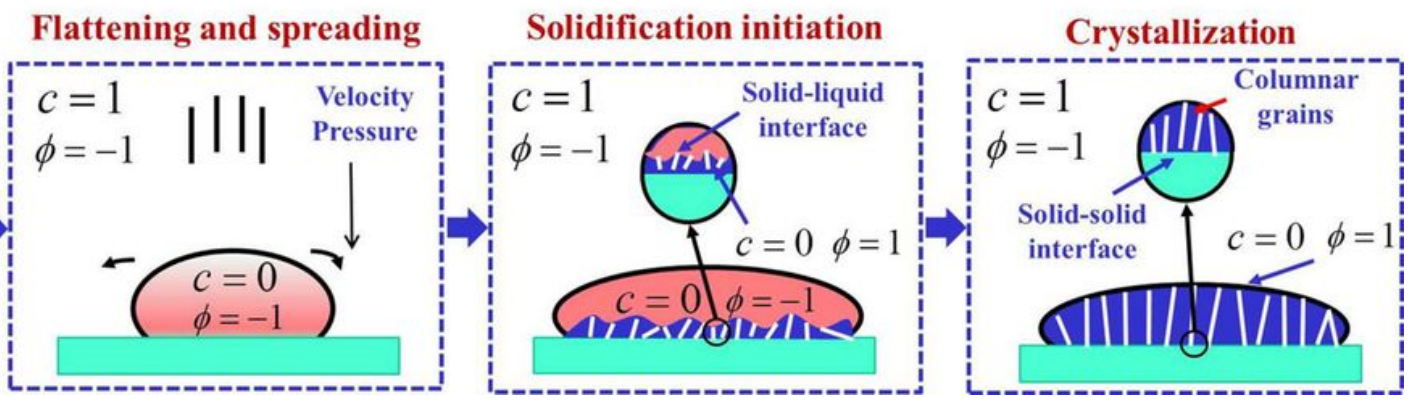

Figure 3

Parameters definition of calculation process: (a) boundary conditions; (b) a modified grid during discretization; (c) order parameter distribution of droplet after impacting on the substrate. 
(a)

$$
\mathbf{n} \cdot \nabla \psi=0
$$

(b)

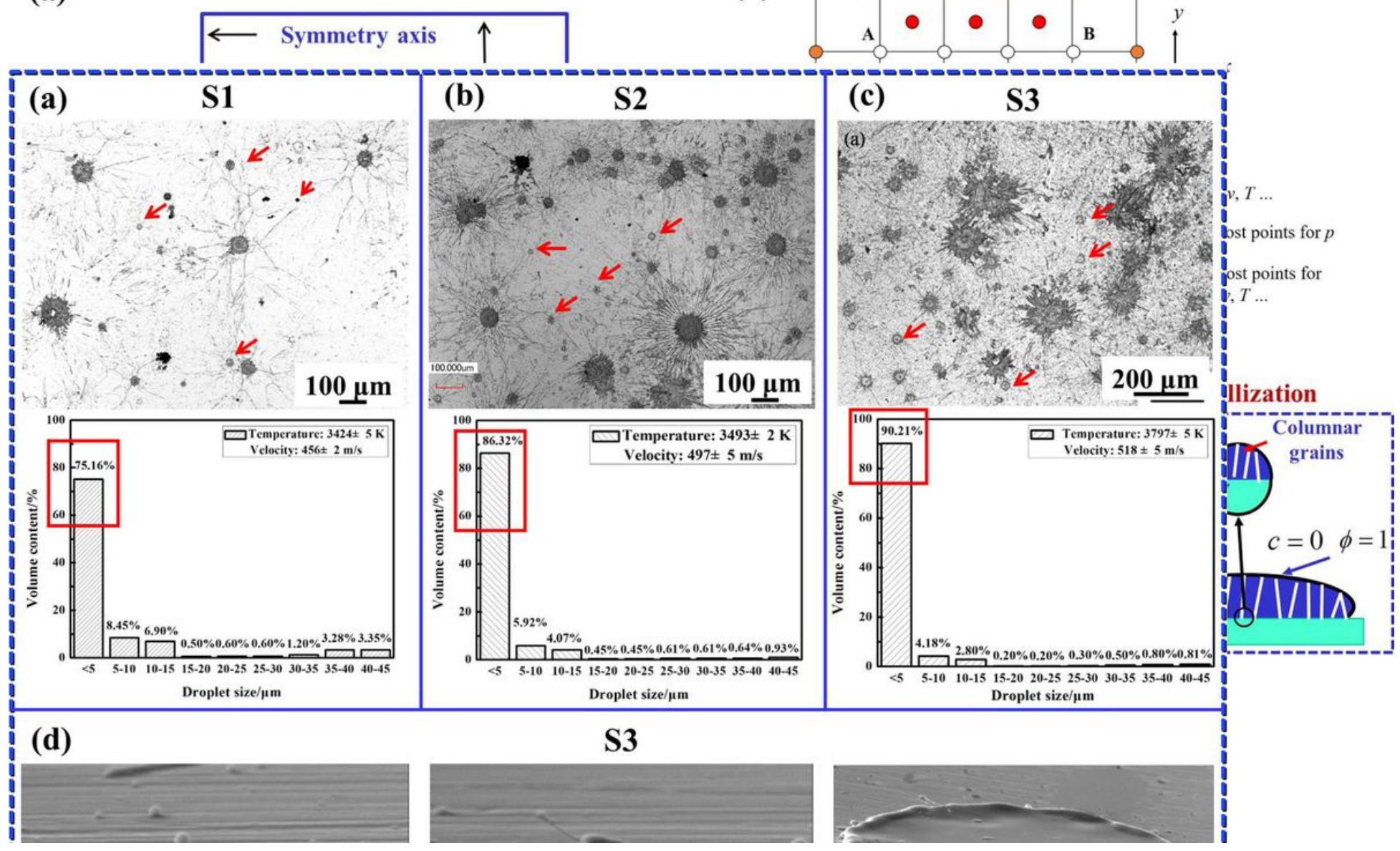

\section{Figure 4}

Morphology and percentage of splats at different in-flight behavior: (a) S1; (b) S2; (c) S3; (d) highmagnificMorphology and percentage of splats at different in-flight behavior: (a) S1; (b) S2; (c) S3; (d) high-magnification images of refinement S3 splat.ation images of refinement S3 splat. 


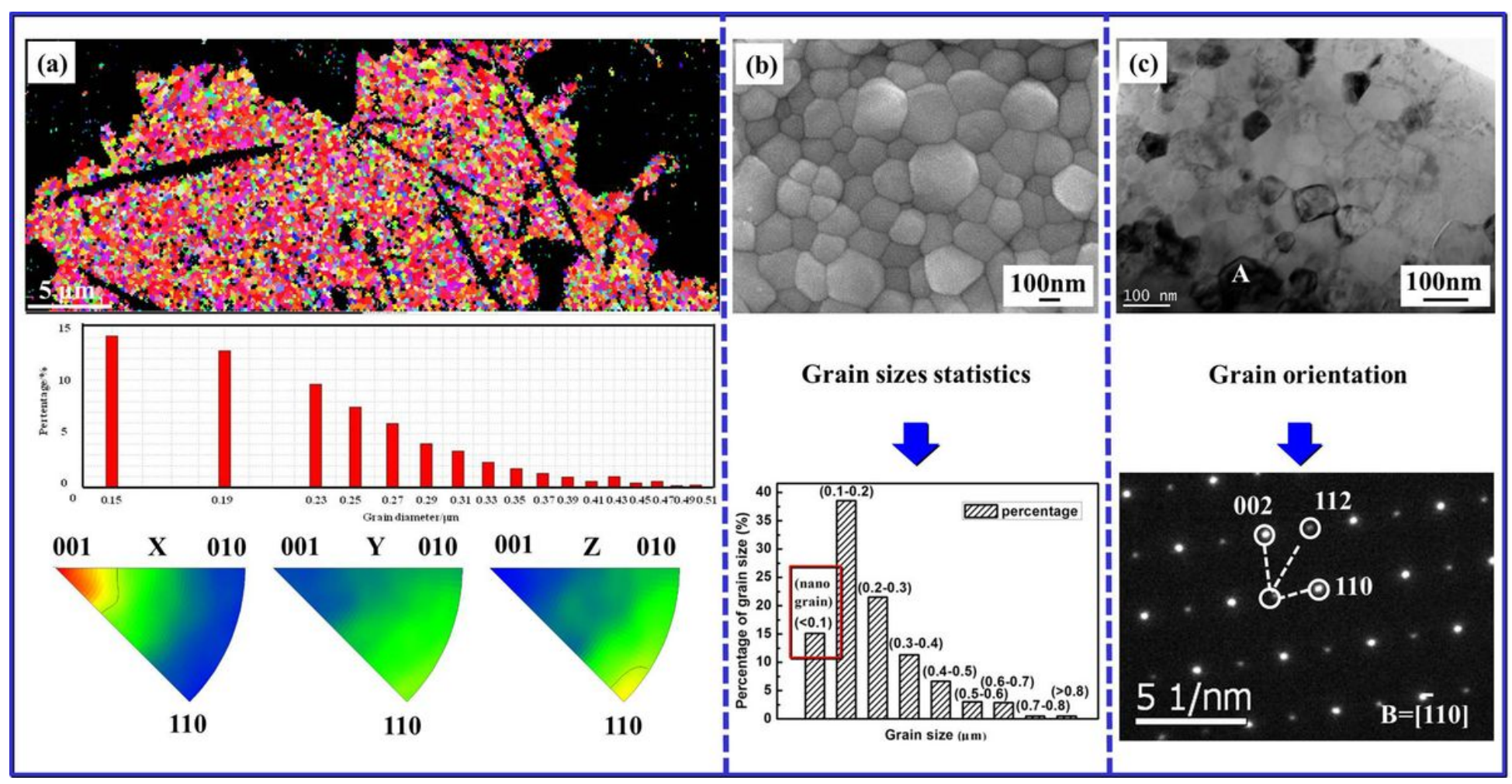

Figure 5

(a) Inverse pole figure (IPF), grain sizes distribution and IPF orientation maps of single-layered splat surface vertical to the substrate (normal direction); (b) Morphology of equiaxed grains and grain sizes statistics within coating after thermal etching at $1250^{\circ} \mathrm{C}$ for $3 \mathrm{~h}$; (c) TEM image of equiaxed grains and corresponding SAED patterns. 
(a)

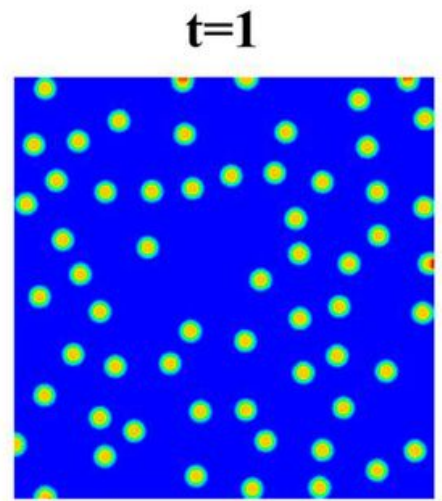

(b)

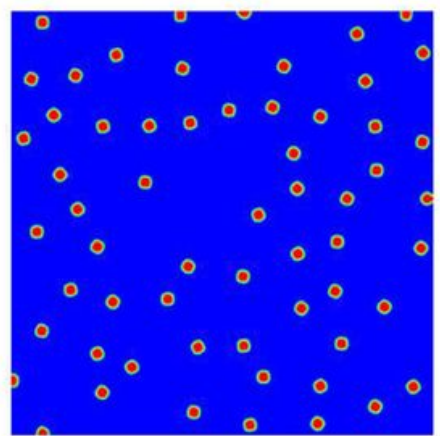

(c)

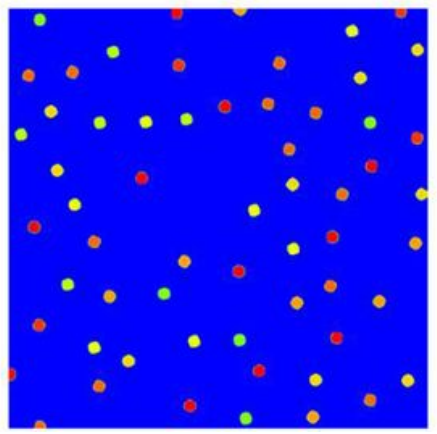

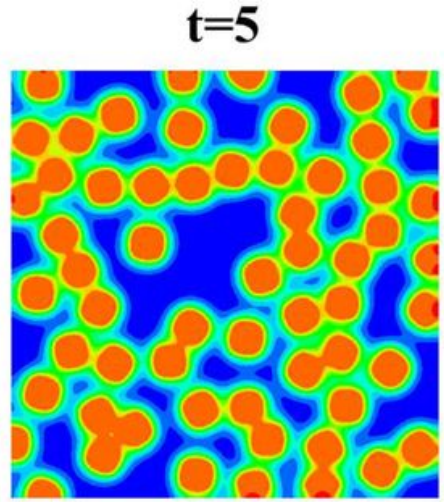
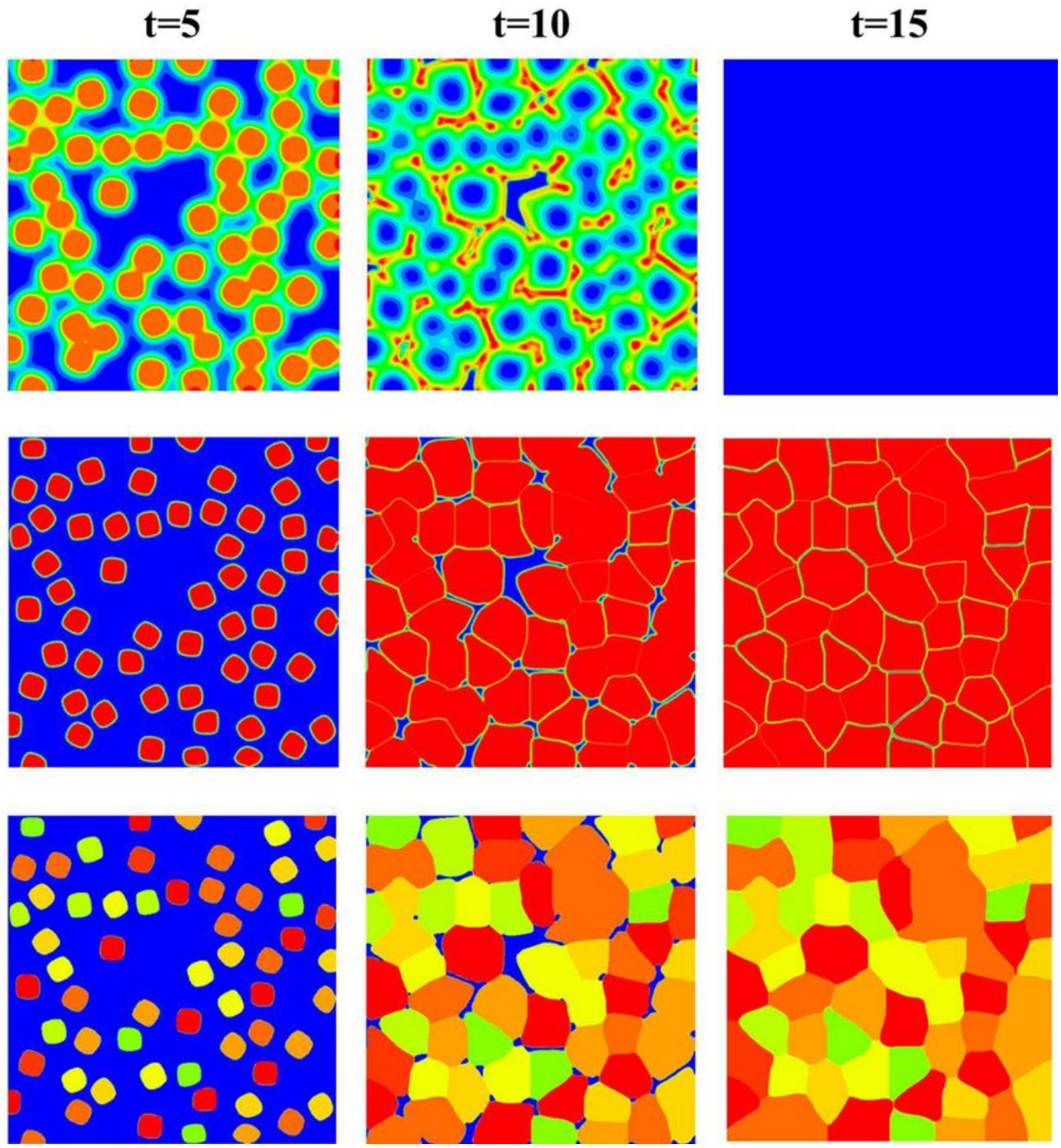

Figure 6

(a) Temperature distribution of equiaxed grains within flattened supersonic droplet during extremely rapid solidification; (b) Growth process of equiaxed grains; (c) Grains orientation at the different times. 
$0.01 \mu \mathrm{s}$
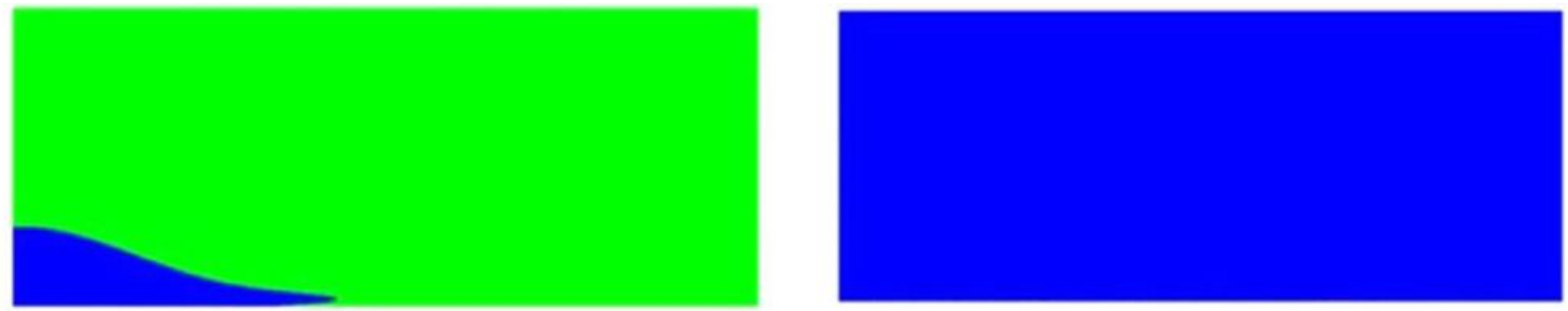

$0.08 \mu \mathrm{s}$
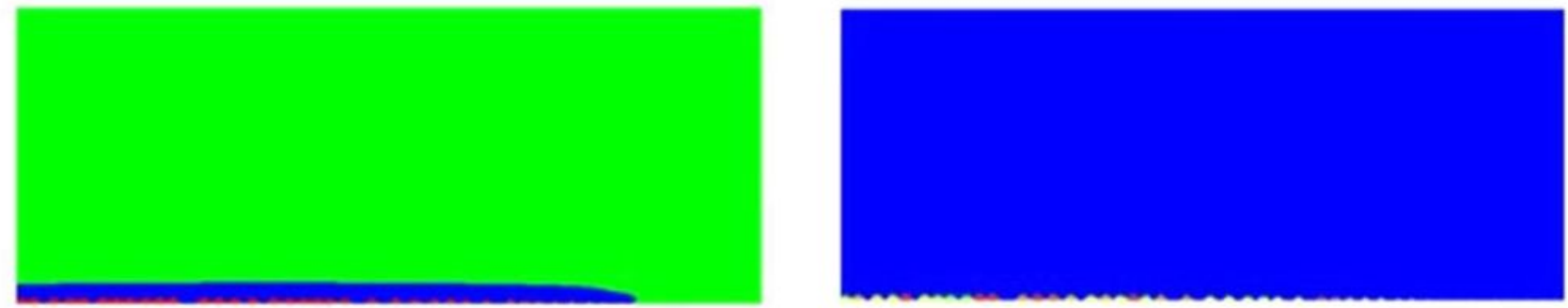

$0.1 \mu \mathrm{s}$
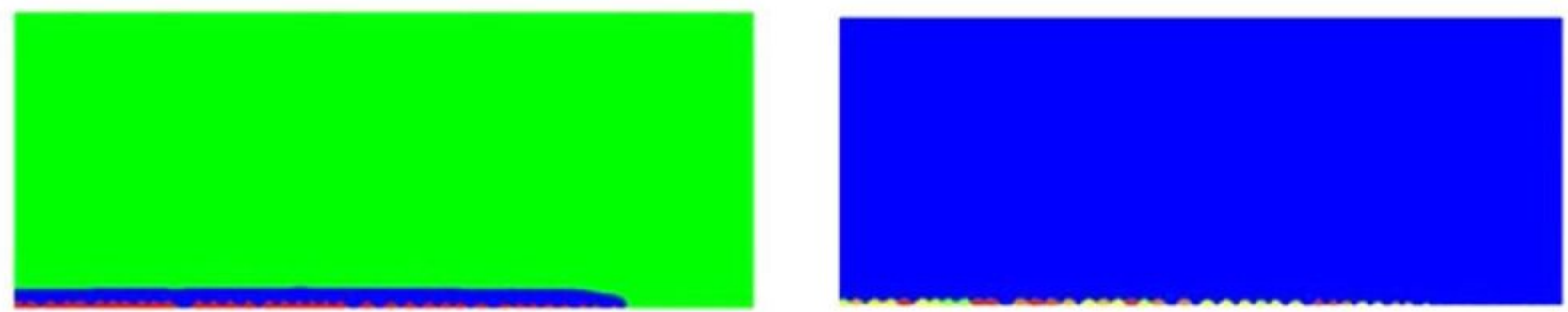

$0.2 \mu \mathrm{s}$
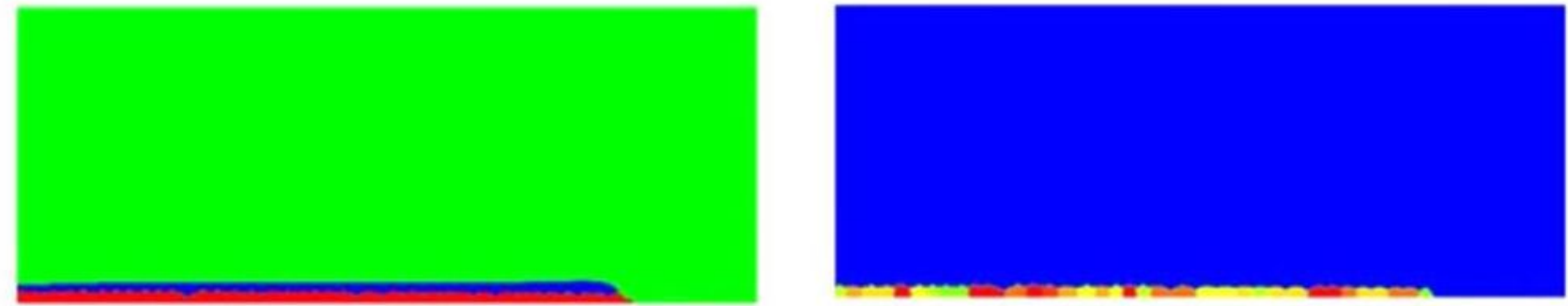

$0.5 \mu \mathrm{s}$
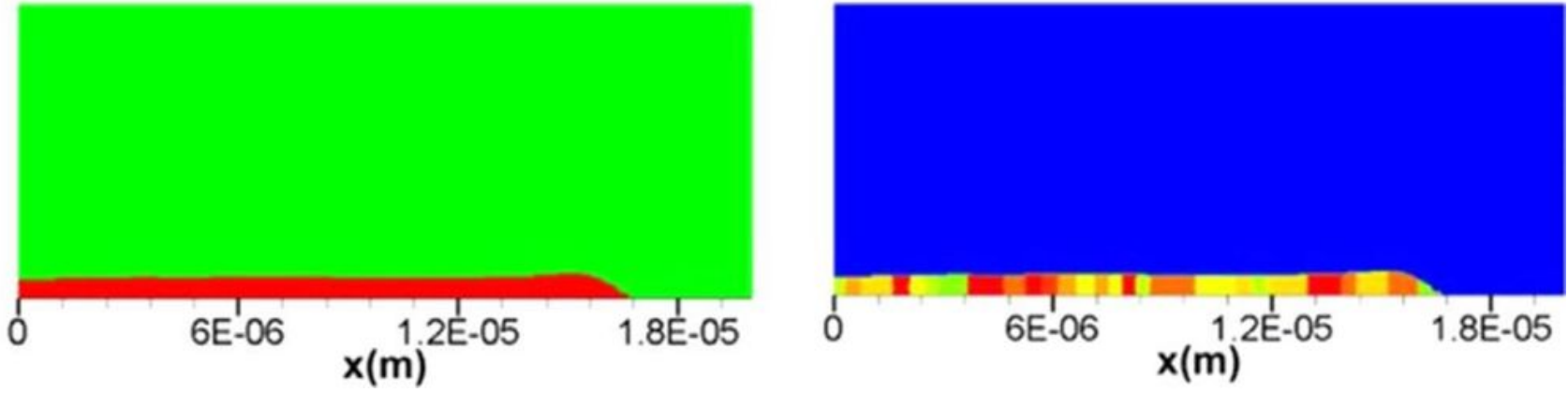

Figure 7

Flattening and solidification with crystallization process of refinement droplet at $518 \mathrm{~m} / \mathrm{s}$. (the green representing gas, the blue liquid, the red solid, respectively, and the different colors indicating different orientations of column grains) 
(a)

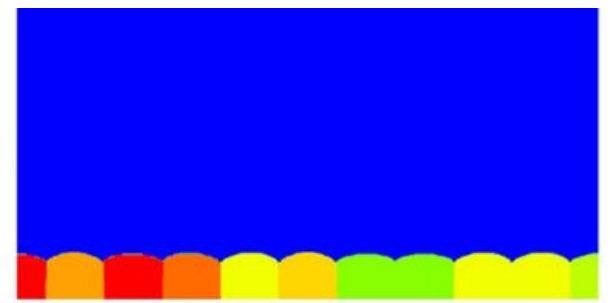

$$
\mathbf{t}=\mathbf{2}
$$

$\mathbf{t}=\mathbf{5}$

(b)

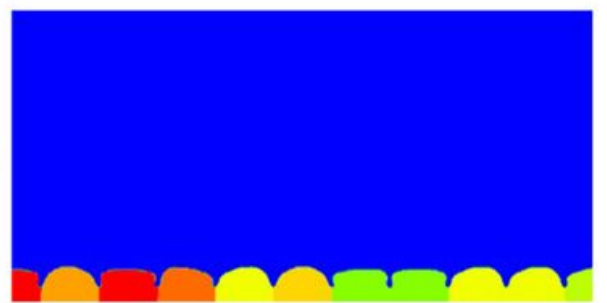

$t=2$

(c)

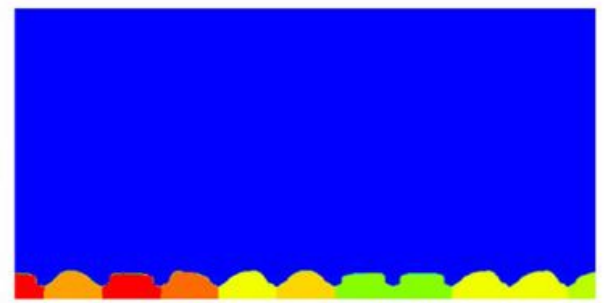

$\mathbf{t}=\mathbf{2}$
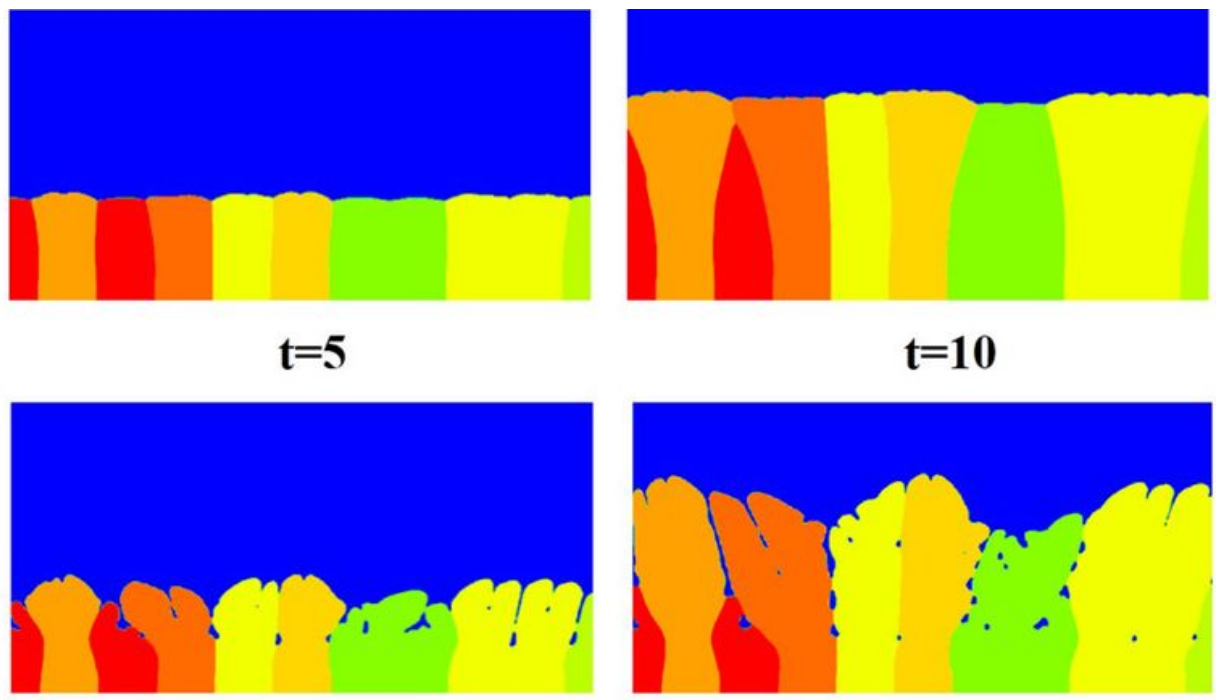

$t=8$
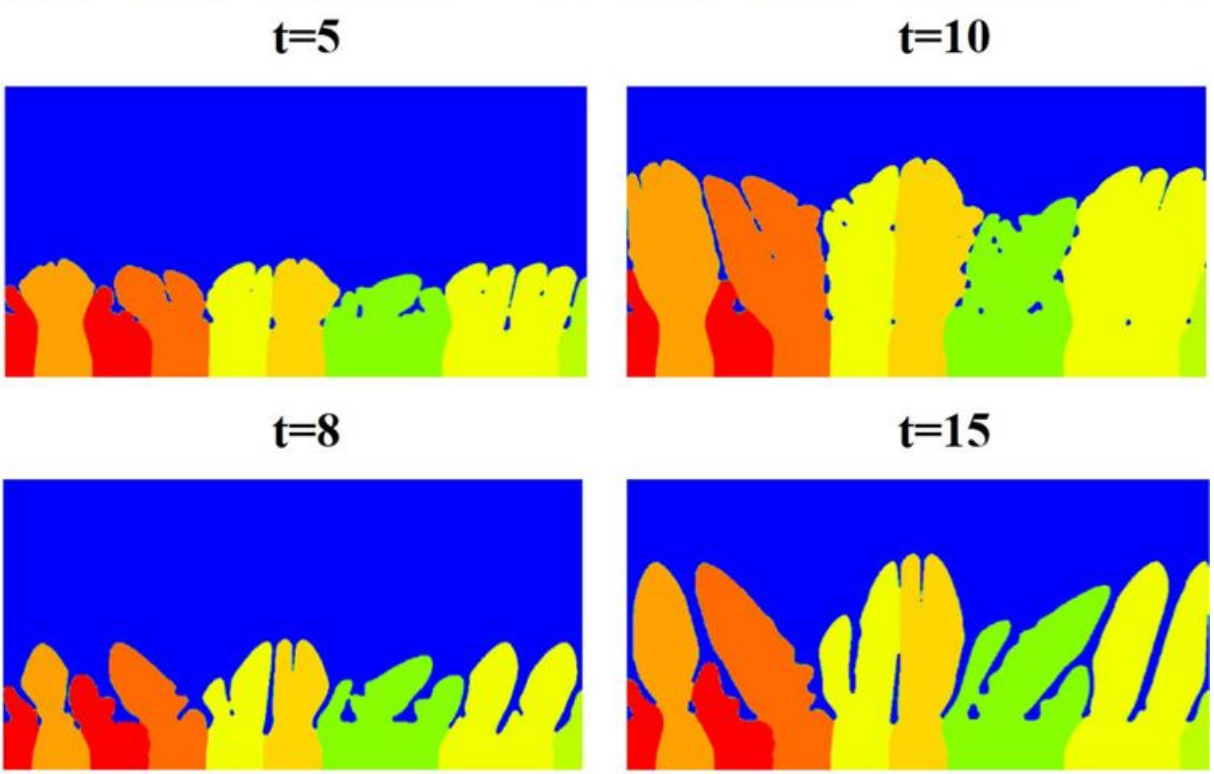

$t=12$

$$
t=15
$$

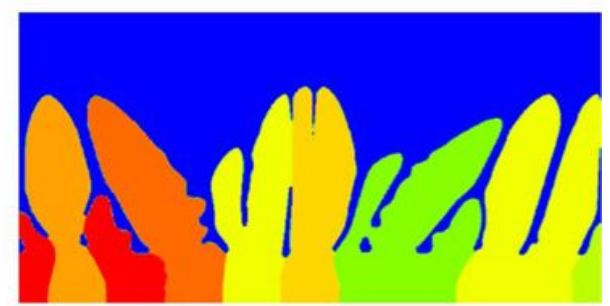

$\mathbf{t}=\mathbf{2 0}$

Figure 8

Competitive growth of columnar grains at different solidification rates: the temperature of bottom surface is (a) $1800 \mathrm{~K}$, (b) $2000 \mathrm{~K},(\mathrm{c}) 2100 \mathrm{~K}$.

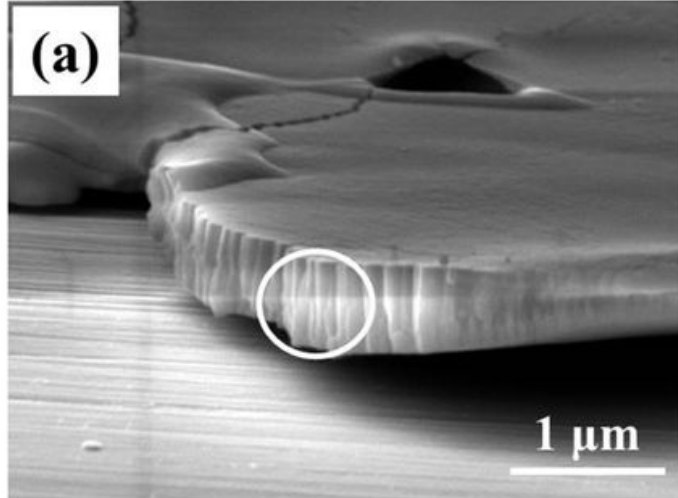

(b)

$0.5 \mu \mathrm{m}$ (c)

$0.01 \mu \mathrm{s}$

$0.1 \mu \mathrm{s}$

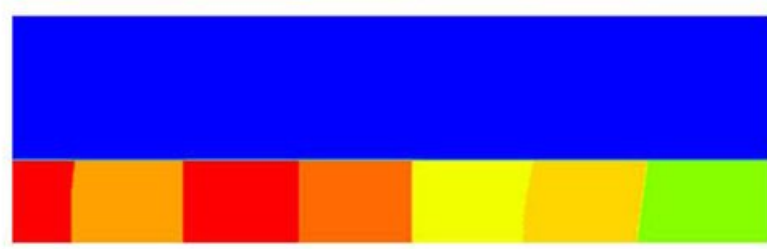

$0.3 \mu \mathrm{s}$

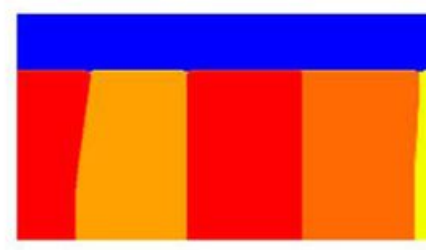

$0.45 \mu \mathrm{s}$

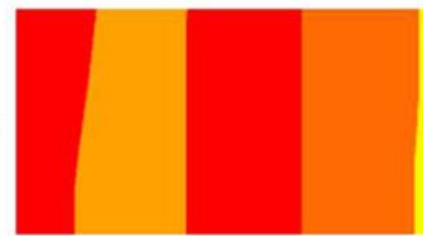

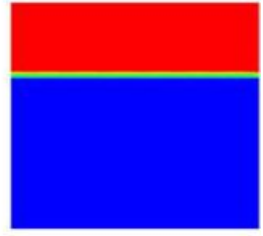
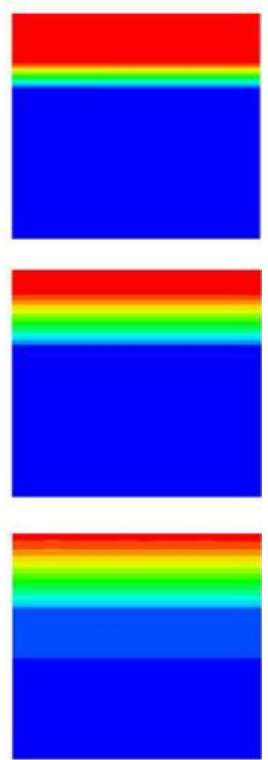


\section{Figure 9}

(a) Columnar grains morphology of YSZ splat at extremely rapid solidification rate; (b) Highmagnification image; (c) Growth behavior and temperature distribution of columnar grains.
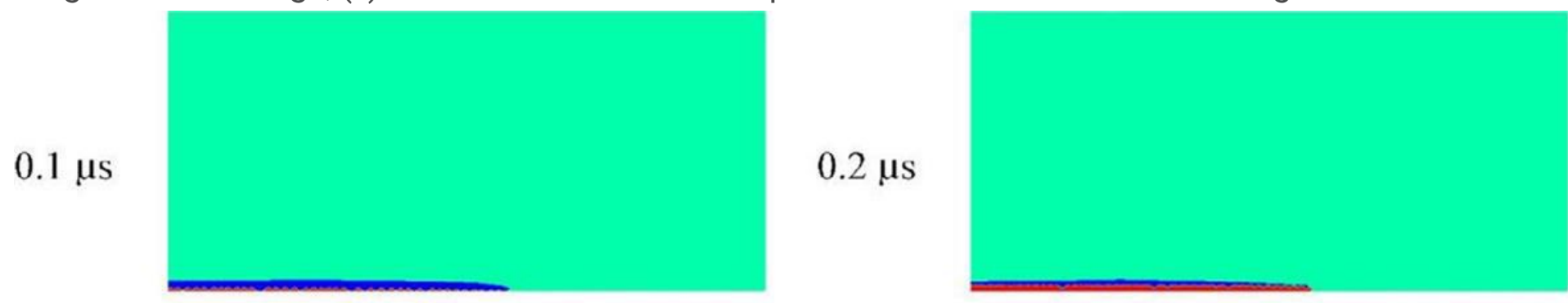

$0.4 \mu \mathrm{s}$

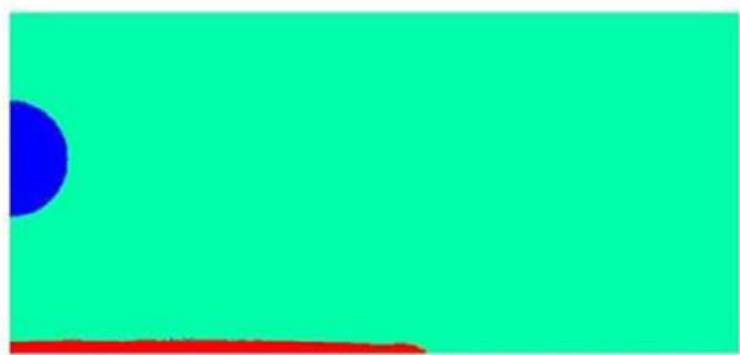

$0.5 \mu \mathrm{s}$

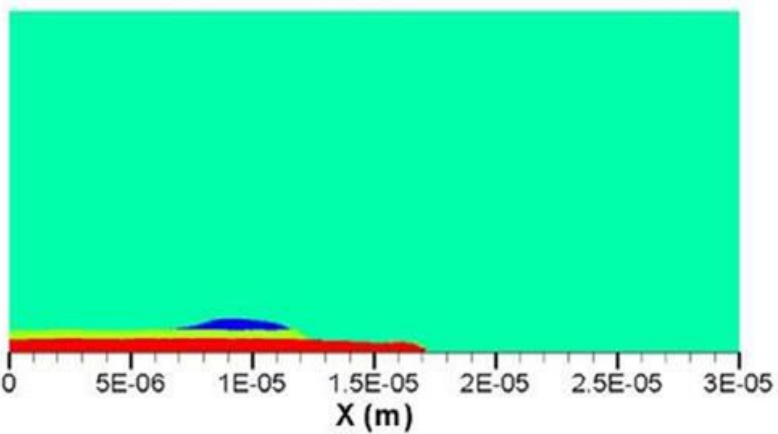

\section{$1.5 \mu \mathrm{s}$}

$0.8 \mu \mathrm{s}$

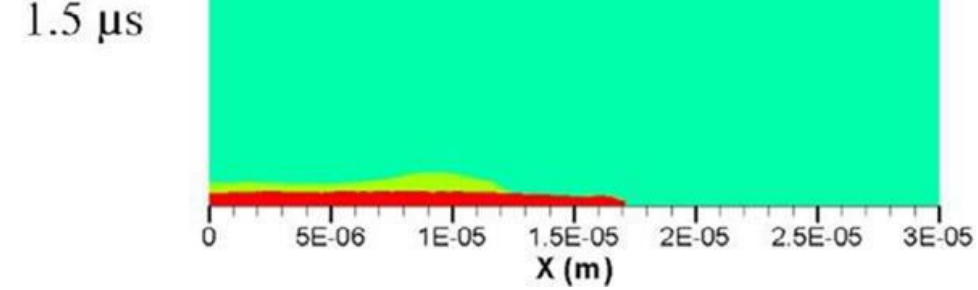

\section{Figure 10}

Flattening and solidification process of two refinement droplets at $518 \mathrm{~m} / \mathrm{s}$. (the green representing gas, the blue liquid and the red solid, respectively) 
(a)

$0.1 \mu \mathrm{s}$

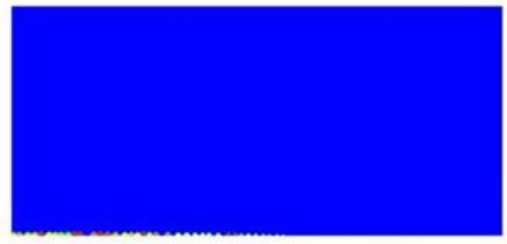

$0.2 \mu \mathrm{s}$

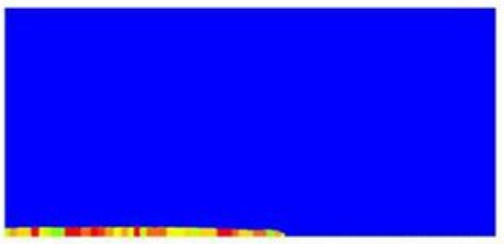

$0.5 \mu \mathrm{s}$

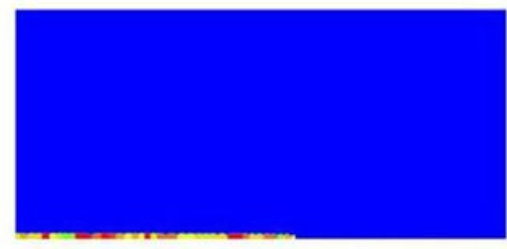

$0.4 \mu \mathrm{s}$

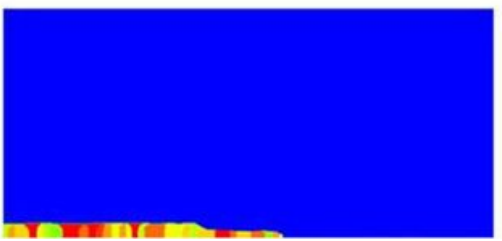

\section{(b)}

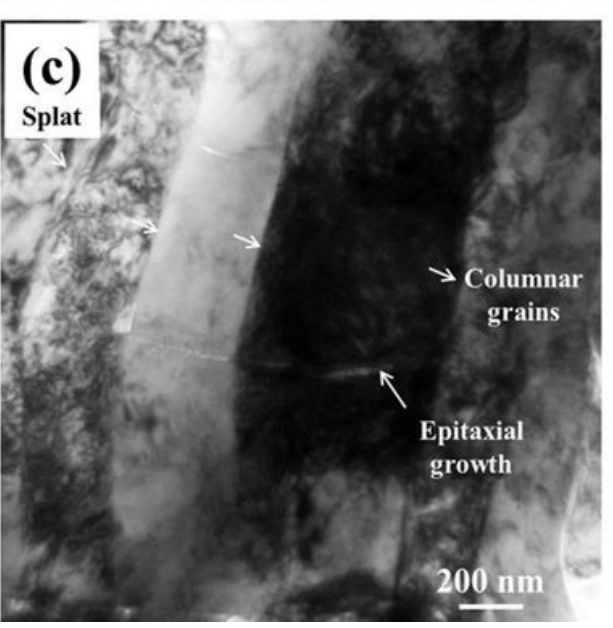

\section{Figure 11}

(a) Growth behavior of columnar grains of two refinement droplets impact on a substrate at $518 \mathrm{~m} / \mathrm{s}$; (b) Simulation results of columnar grains; (c) BF cross-sectional TEM images of columnar grains. 

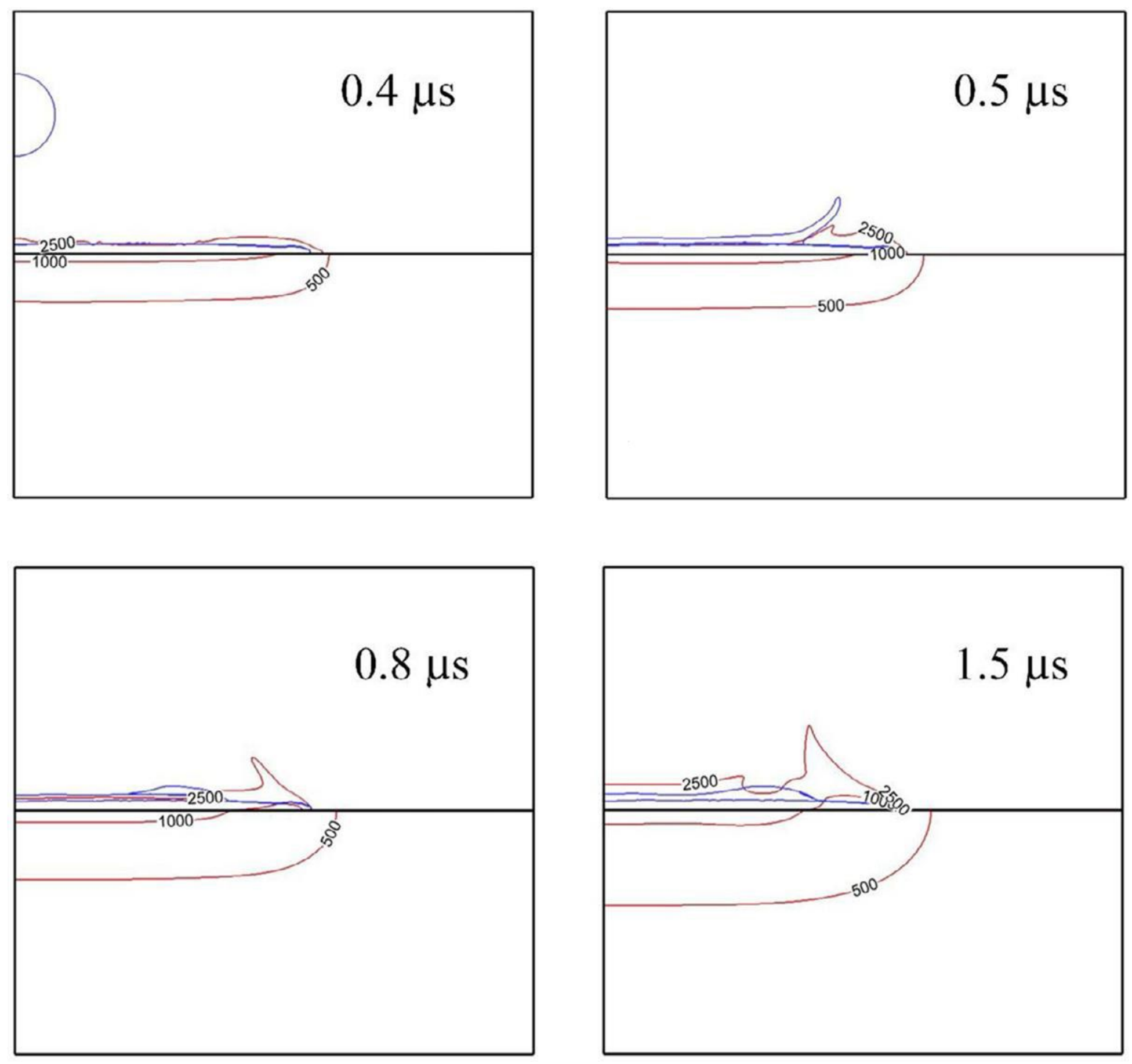

Figure 12

Temperature distribution of sequential refinement droplets impacted on a substrate at $518 \mathrm{~m} / \mathrm{s}$. (The red represents the isothermal line and the blue YSZ droplet) 